\title{
Adaptive control of a wave energy converter
}

\author{
Romain Genest, Josh Davidson and John V. Ringwood, Senior Member, IEEE
}

\begin{abstract}
Energy maximising controllers (EMCs), for wave energy converters (WECs), based on linear models, are attractive in terms of simplicity and computation. However, such (Cummins equation) models are normally built around the still water level as an equilibrium point and assume small movement, leading to poor model validity for realistic WEC motions, especially for the large amplitude motions obtained by a well controlled WEC. The method proposed here is to use an adaptive algorithm to estimate the control model online, whereby system identification techniques are employed to identify a linear model that is most representative of the actual controlled WEC behaviour. Using exponential forgetting, the linear model can be regularly adapted to remain representative in changing operational conditions. To that end, this paper presents a novel adaptive controller based on a receding horizon pseudospectral formulation.

A case study is presented, providing an preliminary evaluation of the adaptive receding horizon pseudospectral control (ARHPC), using simulations based on both linear hydrodynamic modelling and computational fluid dynamics (CFD). The parameter adaptation is shown to behave as expected in both different simulation environments. The ARHPC is observed to perform well in irregular sea states, absorbing more power than its nonadaptive counterpart. The optimal trajectory calculated by the adaptive model is seen to have a smaller motion and power take-off (PTO) forces, compared to those calculated by the nonadaptive linear control model, due to the increased amount of hydrodynamic resistance estimated by the adaptive model, as identified from the nonlinear viscous CFD simulation.
\end{abstract}

Index Terms-Wave energy, adaptive control, system identification, CFD

\section{INTRODUCTION}

The design of EMCs for WECs is challenging, for the following reasons:

1) WECs are correctly described by complex nonlinear equations, which are difficult to parameterize accurately

2) The models which describe WECs vary considerably in structure across different WEC types

3) The resonant motion of controlled WECs challenge small-signal linearisation around a zero displacement equilibrium

Typically for model-based control [1]-[5], a linear WEC model is determined based on Cummins equation [6], with the nonparametric hydrodynamic parameters determined using boundary-element method (BEM) tools. Such models assume small movement around an equilibrium point, corresponding to still water conditions. However, this assumption is challenged, since the ideal WEC behaviour is characterised by significant motion, especially when driven into resonance with the incident waves by the EMC [7], [8]. Furthermore, WEC hydrodynamics are typically characterised by nonlinearities, including viscous damping effects and nonlinear Froude-Krylov forces,

The authors are with the Centre for Ocean Energy Research, Maynooth University, Co. Kildare, Ireland (email:josh.davidson@nuim.ie) where the nature and comparative extent of these nonlinearities vary considerably from device to device [9].

While some nonlinear WEC models have been incorporated into model-based WEC controllers (for example, see [10][12]), nonlinear control solutions are not without their problems. Although a nonlinear model structure is more likely to be a better representation of the true WEC dynamics, the control solution is often difficult, including the required solution of a nonconvex optimisation problem [11], [12].

Considering the desirability of WEC controllers based on linear models, but also the need to capture nonlinear WEC behaviour under controlled conditions, this paper proposes an adaptive controller, which tunes the parameters of a representative linear WEC model, based on measured WEC responses. A receding-horizon pseudospectral controller (RHPC) uses this linear model to determine the optimal velocity trajectory, which maximises the energy capture while observing physical constraints, and a lower-level backstepping controller implements the velocity-following control loop [13]. The adaptive WEC model is initialised with parameters determined from a BEM solver and the model parameters are recursively adapted using a recursive least squares (RLS) algorithm. The parameter updating enables both initial model tuning, as well as continual model adaptation, to ensure relevance to possible changes in the dynamic WEC behaviour due to:

- Changing sea states

- Varying mooring dynamics due to slow drift motions of the WEC and changing tidal elevation [14]

- Marine growth on the WEC

- Green water on the WEC, or water leakage into the WEC

- Non-critical subsystem failure [15]

The adaptation of the control model to remain representative of the actual WEC dynamics, has the potential to increase the controller performance across the variable conditions encountered by a WEC throughout its operational lifetime. [1] suggests that optimal causal control is only a viable approach if the controller parameters adapt to changes in the sea state, showing the necessity of gain-scheduling in accordance with changes in the spectral content and propagatory direction of the sea state. A comparison of selected adaptive control strategies for WECs, such as gain scheduling and extremumseeking adaption, is presented in [4]. A review of adaptive control employed for wave energy applications is given in [16].

As a case study, this paper demonstrates the implementation of the adaptive controller in numerical wave tank (NWT) simulations. The controller operates interactively with the simulation and updates its internal model, using system identification techniques, based on measured outputs [17]. This has evolved from earlier work, where fixed hydrodynamic models are identified using measured responses from NWT experiments 
[18]-[20]. The present adaptive control case extends this by identifying the models online within the NWT simulation.

\section{THE ADAPTIVE CONTROLLER}

The adaptive controller is an extension of the RHPC control detailed in [13]. A critical comparison of the RHPC and traditional model predictive control (MPC) for wave energy devices is given in [21], revealing that both approaches have comparable performances, but that the RHPC typically requires a third of the computational expense. The RHPC is extended in the present work by allowing the linear control model to adapt to the measured WEC responses.

The linear control model is detailed in Section II-A, and then Section II-B describes the RLS adaptive algorithm employed to update the parameters of the linear control model online, based on measurements of the WEC behaviour. Section II-C details the optimal controller, which utilises the linear control model to calculate the optimal WEC velocity trajectory to maximise energy absorption while observing path constraints.

\section{A. Linear control model}

The adaptive control model is a linearised version of Newton's second law of motion applied to the WEC, based on Cummins' equation [6]:

$\left(m+\mu_{\infty}\right) \frac{d v(t)}{d t}+\int_{0}^{t} K_{r}(t-\tau) v(\tau) d \tau+S_{h} x(t)=F_{e x}(t)+u(t)$

Here, $m$ and $\mu_{\infty}$ are the mass and the infinite frequency added mass of the system, $K_{r}(t)$ is the kernel function involved in a convolution product with the velocity, representing a linearised version of the radiation force, $S_{h}$ is the hydrostatic stiffness, while $F_{e x}(t)$ is a linearised expression of the excitation force generated by unperturbed incoming waves onto the WEC's hull, at its equilibrium position. $x(t)$ and $v(t)$ are the system position and velocity, respectively, representing the state variables, and $u(t)$ is the control force applied by a power take off (PTO) system.

1) Pseudospectral representation: The state and control variables are approximated by a truncated series of orthogonal basis functions on a fixed control horizon, $I=\left[t, t+T_{0}\right]$, where $T_{0}$ the control horizon over which the energy absorption is maximised:

$$
\forall t \in I, f(t) \approx f^{N}(t)=\sum_{i=1}^{N} \tilde{f}_{i} \phi_{i}(t)=\boldsymbol{\Phi}(t) \tilde{\mathbf{f}}
$$

$f^{N}(t)$ is a truncated series that approximates the initial function, $f(t)$ (which could be a state or control variable), as a finite sum of weighted basis functions, $\boldsymbol{\Phi}(t)=\left\{\phi_{i}(t)\right\}_{i=1}^{N}$. The vector, $\tilde{\mathbf{f}}=\left[\tilde{f}_{1}, \ldots, \tilde{f}_{N}\right]^{T}$, contains the projections of $f(t)$ onto the basis functions:

$$
\tilde{f}_{i}=\int_{I} \phi_{i}(t) f(t) d t
$$

2) Basis functions: For WEC optimal control problems, Fourier-type bases seem to be a straightforward choice, since the harmonic wave elevation and hydrodynamic forces are usually well described via Fourier analysis. However, real-time control and finite-time control horizons require approximations of nonperiodic functions. Therefore, in the present work, halfrange Chebyshev Fourier (HRCF) functions, which provide a Fourier extension for nonperiodic signals, are selected for the bases. The HRCF functions are defined in [22], and employed in a RHPC in [13]. Choosing $n$ frequencies leads to $N=2 n+1$ basis functions, with $n$ sine, $n$ cosine and one constant term, shown in Fig. 1 for $n=4$.
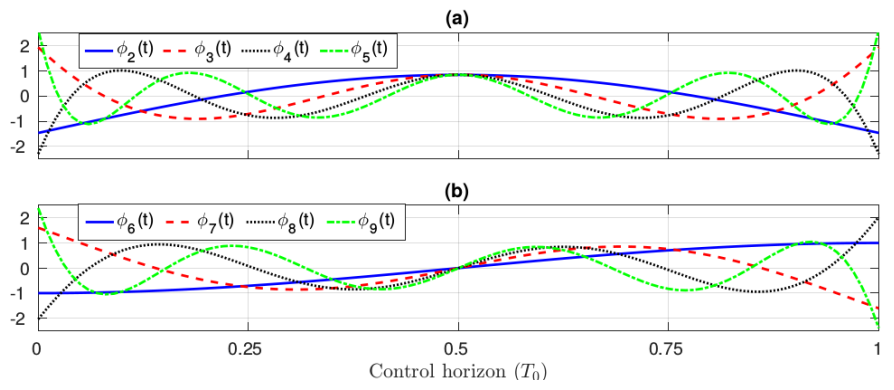

Fig. 1. The first four frequencies of the (a) cosine $(\Phi[2: n+1])$ and (b) sine $(\boldsymbol{\Phi}[n+2: 2 n+1])$ HRCF basis functions. The first basis function is a constant: $\phi_{1}(t)=\sqrt{1 / 2}$

3) Receding horizon pseudospectral control model: Cummins' equation (Eqn. (1)), is expressed in terms of state and control variable projections ( $\tilde{\mathbf{x}}, \tilde{\mathbf{v}}$ and $\tilde{\mathbf{u}}$ ), and is evaluated over the control horizon, at $N$ collocation points, $t_{k}$, as:

$$
\boldsymbol{\Phi}\left(t_{k}\right)\left([\mathbf{M}, \mathbf{N}]\left[\begin{array}{c}
\tilde{\mathbf{x}} \\
\tilde{\mathbf{v}}
\end{array}\right]-\tilde{\mathbf{u}}\right)=F_{e x}\left(t_{k}\right)-F_{r}\left(t_{k}\right)
$$

where $\mathbf{M}$ and $\mathbf{N}$ are $N \times N$ matrices, that multiply by the truncated series approximations of the corresponding state variable, to represent the position and velocity dependent forces in Eqn. (1), respectively. The term $F_{r}(t)$, is the radiation force generated by past values of the velocity affecting the control horizon, whereby the radiation convolution product in Eqn. (1) is decomposed into two parts, depending either only on past or future values of the device velocity. The radiation force depending on future values of the velocity is then included inside the operator matrix $\mathbf{N}$. An example showing the elaboration of Eqn. 4 in detail is given in [13].

By developing the linear equation of the system, we obtain:

$$
\sum_{i} \sum_{j} m_{i j} \tilde{x}_{i} \phi_{j}\left(t_{k}\right)+\sum_{i} \sum_{j} n_{i j} \tilde{v}_{i} \phi_{j}\left(t_{k}\right)=F\left(t_{k}\right)
$$

where $F(t)=u(t)+F_{\text {ex }}(t)-F_{r}(t)$. The two double sums can be seen as a projection of the position and velocity onto given kernel functions, $m$ and $n$, respectively, such that:

$$
\begin{aligned}
\sum_{i} \sum_{j} m_{i j} \tilde{x}_{i} \phi_{j}(t) & =\int_{I} m^{N}(t, \tau) x^{N}(\tau) d \tau \\
\sum_{i} \sum_{j} n_{i j} \tilde{v}_{i} \phi_{j}(t) & =\int_{I} n^{N}(t, \tau) v^{N}(\tau) d \tau
\end{aligned}
$$


where $m^{N}(t, \tau)=\sum_{i} \sum_{j} m_{i j} \phi_{i}(\tau) \phi_{j}(t)$ and $n^{N}(t, \tau)=$ $\sum_{i} \sum_{j} n_{i j} \phi_{i}(\tau) \phi_{j}(t)$. The equation of motion is then transformed in a Fredholm integral equation of the first kind, as:

$$
\int_{I} m^{N}(t, \tau) x^{N}(\tau) d \tau+\int_{I} n^{N}(t, \tau) v^{N}(\tau) d \tau=F(t)
$$

4) Model initialisation: From Eq. (1), the initial kernel functions $m_{0}^{N}$ and $n_{0}^{N}$ can be written as:

$$
\begin{aligned}
m_{0}^{N}(t, \tau) & =S_{h} \delta(t-\tau) \\
n_{0}^{N}(t, \tau) & =\left(m+\mu_{\infty}\right) \dot{\delta}(t-\tau)+K_{r}(t-\tau)
\end{aligned}
$$

where $\delta$ and $\dot{\delta}$ correspond, respectively, to the Dirac delta function and its first derivative. This illustrates the ability of the model formulation to represent the standard Cummins equation. The control model parameters are initialized from Eqs. (9) and (10) , using hydrodynamic parameter values, $S_{h}, \mu_{\infty}$ and $K_{r}(t)$, obtained from a BEM solver.

\section{B. Parameter adaptation}

The matrix $[\mathbf{M}, \mathbf{N}]$, is updated at each $T_{R L S}$ time-step, $p$, using a standard RLS algorithm [23], which tries to obtain the best kernel functions, $m^{N}$ and $n^{N}$ from Eq. (8), to satisfy the motion of the system, using the following recursive algorithm:

$$
\begin{aligned}
\theta & =\left[\tilde{\mathbf{x}}^{T}, \tilde{\mathbf{v}}^{T}\right]^{T} \\
\mathbf{e}(p) & =\mathbf{d}(p)-\theta^{T}[\mathbf{M}, \mathbf{N}]^{T}(p-1) \\
\mathbf{r}(p) & =\mathbf{P}(p-1) \theta /\left(\lambda+\theta^{T} \mathbf{P}(p-1) \theta\right) \\
\mathbf{P}(p) & =\lambda^{-1}\left(\mathbf{P}(p-1)-\mathbf{r}(p) \theta^{T} \mathbf{P}(p-1)\right) \\
{[\mathbf{M}, \mathbf{N}](p) } & =[\mathbf{M}, \mathbf{N}](p-1)+\mathbf{e}(p) \mathbf{r}(p)
\end{aligned}
$$

where the covariance matrix $\mathbf{P}$ is initialised as $\mathbf{P}(0)=p_{0} \mathbf{I}$ (where $\mathbf{I}$ is the identity matrix). The vector $\mathbf{d}$ contains the measured outputs the linear model should emulate, which in this case is $\tilde{\mathbf{F}}$, the projection of $F(t) . \lambda$ is the forgetting factor, allowing a self-tuning $(\lambda=1)$ or adaptive control $(0<\lambda<1)$.

\section{Optimal control}

The optimal control consists of two layers. The first layer calculates the optimal trajectory the WEC should follow for energy maximisation, while observing path constraints (typically amplitude and/or force limitations). The second layer controls the PTO force to ensure the WEC follows the optimal trajectory prescribed by the first layer.

1) Optimal trajectory calculation: The optimal controller maximises the absorbed energy, $J$, over the control horizon:

$$
J=-\int_{I} v(t) u(t) d t
$$

Replacing $v(t)$ and $u(t)$ with their truncated series:

$$
J \propto-\tilde{\mathbf{v}}^{T} \tilde{\mathbf{u}}
$$

Since the basis functions are orthogonal, the cost function is directly proportional to the sum of the product of $\tilde{v}_{i}$ and $\tilde{u}_{i}$, which leads to a convex optimisation problem; a strength of this linear control formulation.
While maximising $J$, the control algorithm must also ensure that the state and control variables obey the dynamic equations describing the WEC behaviour. Expressed in terms of residuals, and replacing state and control variables in Eqn. (4)by the truncated series, we obtain the following linear equations:

$$
\begin{aligned}
r_{1}(t)= & \boldsymbol{\Phi}(t)[\mathbf{D} \tilde{\mathbf{x}}-\tilde{\mathbf{v}}] \\
r_{2}(t)= & \boldsymbol{\Phi}(t)\left[\left(\left(m+\mu_{\infty}\right) \mathbf{D}+\mathbf{R}\right) \tilde{\mathbf{v}}+S_{h} \tilde{\mathbf{x}}-\tilde{\mathbf{u}}\right] \ldots \\
& +F_{r}(t)-F_{e x}(t)
\end{aligned}
$$

where $\mathbf{D}$ is the differention matrix defined in [24], $\mathbf{R}$ is the radiation matrix defined in [13], corresponding to the radiation force generated by the velocity over the control horizon $I$.

Path constraints, such as PTO force or position excursion limits, can be easily taken into account by the RHPC algorithm, whereby the controller determines a velocity setpoint which is within the physical displacement/PTO force constraints of the system. For example, in order to avoid any slamming phenomenon that could occur if the device comes out of the water, as can happen when applying complexconjugate control without position constraints, the relative position of the body with respect to the actual free surface, $\eta(t)$, is constrained to be smaller than a given geometrical parameter $H$,

$$
|\boldsymbol{\Phi}(t) \tilde{\mathbf{x}}-\eta(t)| \leq H .
$$

The control algorithm needs to maximise the cost function $J$, bring the residuals $r_{1}$ and $r_{2}$ to zero, and ensure the satisfaction of linear inequality constraints. The optimisation problem is quadratic, allowing the use of efficient optimisation techniques, and is re-solved at a regular time-steps, $\Delta t_{R H P C}<I$, following the receding horizon approach [25]. For more details on the RHPC algorithm, the reader may refer to [13] and [21].

2) Trajectory tracking: A backstepping method is implemented for trajectory tracking, forcing the nonlinear dynamical WEC system to follow the optimal trajectory calculated by the linear control model. Backstepping, employing feedback linearisation, is shown to have good robustness properties [26] and follows the general robust hierarchical structure in [27]. The implementation of the backstepping method is described in [13], where it is shown to minimise the position error, $e_{2}(t)$, and the velocity error, $e_{2}(t)$ :

$$
\begin{aligned}
& e_{1}(t)=x(t)-x_{\text {ref }}(t) \\
& e_{2}(t)=v(t)-v_{\text {ref }}(t)
\end{aligned}
$$

where $x_{r e f}(t)$ and $v_{r e f}(t)$ are the reference position and velocity trajectories, respectively.

The PTO force, $F_{P T O}$, that ensures the WEC follows the calculated reference trajectory, is given by:

$$
\begin{aligned}
F_{P T O}(t)= & -\left(m+\mu_{\infty}\right)\left(e_{1}(t)-\ddot{x}_{d}(t)+\tau_{2} e_{2}(t)\right) \ldots \\
& +S_{h} x(t)+\int_{0}^{t} K_{r}(t-\tau) v(\tau) d \tau-F_{e x}(t)
\end{aligned}
$$

where $\ddot{x}_{d}(t)$ is the time derivative of $\dot{x}_{d}(t)=v_{\text {ref }}(t)-\tau_{1} e_{1}(t)$, which is an intermediate variable related to the desired velocity, and $\tau_{1}$ and $\tau_{2}$ are constants (in units of $s^{-1}$ ) defining the rate of convergence of $e_{1}(t)$ and $e_{2}(t)$. 


\section{CASE STUdY}

The performance of the ARHPC is assessed here using numerical simulations. The simulation structure is detailed in Section III-A. The two different simulation environments used in the assessment are described in Section III-B. A description of the test case considered is outlined in Section III-C, and the results presented in Section III-D.

\section{A. Simulation structure}

The global architecture of the control algorithm, and its simulation environment, is shown in Fig.2. The control algorithm can be decomposed into three distinct stages, each running with individual time-steps:

- The backstepping controller (Green) is contained as a routine within the simulation, and updates the control force at each time step $\Delta t_{\text {Sim }}$. The input reference trajectory is defined by its projections onto the orthogonal set of basis functions, and thus can be easily estimated at any given instant without the need for interpolation.

- The RHPC (Red) computes the reference trajectory iteratively at a regular time-step, $\Delta t_{R H P C}$. The solution of the reference trajectory projection maximises the energy absorption, while ensuring path constraints. The optimisation problem is solved using the active-set algorithm in the quadprog function in MATLAB.

- The adaptive algorithm (Blue) updates the linear control model at a regular time-step $\Delta t_{R L S}$. The updated control model is then stored and used by the RHPC to find the reference trajectory. The choice of $\Delta t_{R L S}$ is important, in that it must be chosen short enough to allow good tracking of changes in a linear representative model corresponding to sea state variations, while not so short as to attempt instantaneous tracking of the nonlinearities.

The excitation force (White) must be estimated over the future control horizon $T_{0}$. A comparison of forecasting metodologies is presented in [28], which concludes that very accurate predictions of swell waves can be obtained up to two wave periods into the future. In the present paper, the effect of the excitation force prediction is removed, and the ARHPC is evaluated under the assumption of perfect knowledge of the incident wave series.

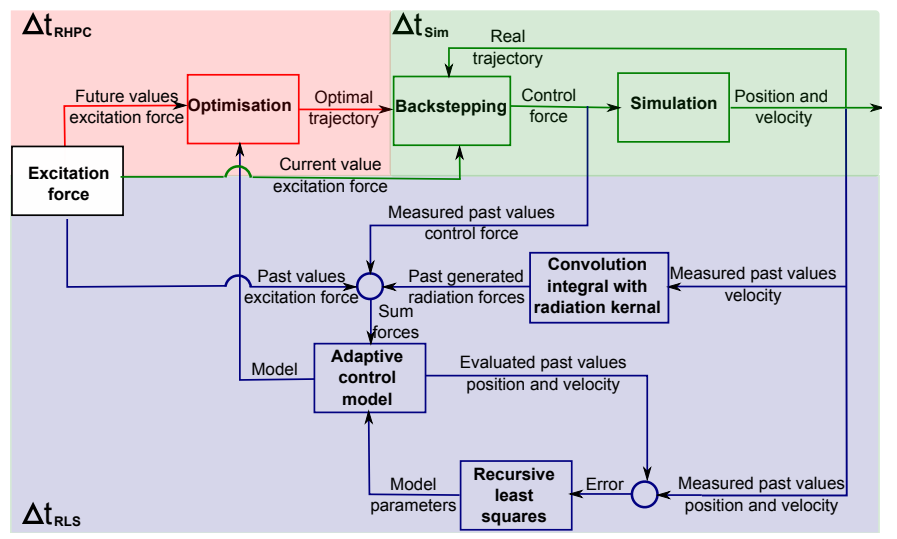

Fig. 2. Global architecture of the control algorithm and the WEC simulation

\section{B. Simulation environments}

The study employs both linear hydrodynamic model and CFD-based numerical wave tank (NWT) simulations as evaluation tools for the adaptive WEC controller.

1) Linear simulation: The linear hydrodynamic model yields fast simulations, allowing the evolution of the adaptive model parameters to be investigated over many wave periods or sea states. Additionally, the parameters in the linear hydrodynamic model can be easily changed to specific values, to assess the accuracy of corresponding changes in the adaptive model. The linear model is implemented in MATLAB, and employs a 4th order Runge-Kutta (R-K) scheme to solve Cummins equation (Eqn. 1), using constant time steps $\Delta t_{\text {Sim }}=10^{-3} \mathrm{~s}$. The hydrodynamic parameters are obtained from the open source linear potential theory BEM software Nemoh [29]. It should be noted that the R-K disretisation differs significantly from the pseudospectral model parameterisation used in the control calculations.

2) CFD NWT simulation: The CFD simulation environment requires intensive computation and large run-times; however, the CFD NWT evaluation ensures the maximum fidelity in the calculation of the WEC response, capturing important nonlinear hydrodynamic effects, such as viscous damping and nonlinear Froude-Krylov forces, which have pronounced relevance for a controlled WEC [7], [8]. The CFD NWT provides a realistic simulation model which is different from the control model, allowing the convergence and adaptive properties of the present control scheme to be tested, where the ARHPC will try to create the best representative linear control model corresponding to the conditions encountered in the nonlinear simulation. The NWT is implemented using the open source CFD software, OpenFOAM, as detailed in [30], and already utilised for WEC control evaluation in [8], [31]. Full details of the CFD NWT simulation of the present ARHPC are described in [16]. The CFD simulation uses an adaptive timestep to ensure the Courant condition is satisfied, leading to timesteps typically $10^{-3} s>\Delta t_{\text {Sim }}>10^{-4} s$.

\section{Test case description}

The case study considers a point absorber WEC, comprising a spherical buoy, constrained to heave motion only, equipped with an ideal PTO capable of providing/extracting bidirectional power to/from the heaving buoy, see Fig.3. The mass density of the WEC is half of the water density, so that the sphere is $50 \%$ submerged at equilibrium. The sphere has a $0.1 \mathrm{~m}$ radius and $0.61 \mathrm{~s}$ natural period, representing a scaled down version of a realistic WEC, chosen to reduce the required $\mathrm{CFD}$

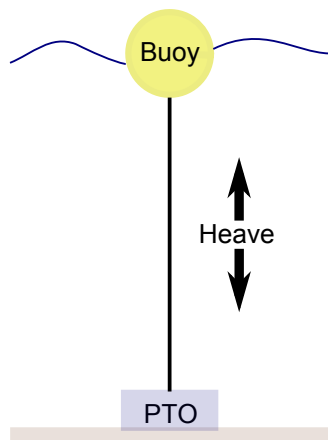

Inertia (Sea floor/damper plate)

Fig. 3. Case study WEC computation time as discussed in [16], [32]. This test case is chosen identical to that presented in [8], [16]. 
1) Input waves: An input wave series representing a JONSWAP spectrum, with a peak period of $1 s$, is employed (see Fig. 4). Note the peak period differs from the natural period of the WEC, which is $0.61 s$.

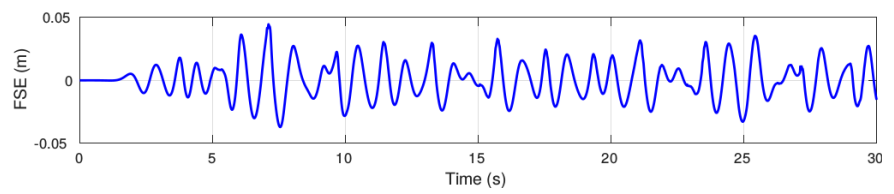

Fig. 4. First $30 \mathrm{~s}$ of the input wave series used for the test case.

2) Model initialisation: Choosing seven frequencies for the RHPC basis functions was found to give a parsimonious balance between accuracy and complexity in approximating the solution. The linear control model therefore comprises two $15 \times 15$ submatrices, $\mathbf{M}$ and $\mathbf{N}$. Using hydrodynamic parameters calculated from the BEM solver Nemoh, the parameter values for $\mathbf{M}$ and $\mathbf{N}$ are initialised using Eqs. (9) and (10), respectively, and are displayed in Fig. 5.

- The matrix $\mathbf{M}$ is initialised via: $\mathbf{M}=S_{h} \mathbf{I}$.

$\mathbf{M}$ appears in Fig. 5-(a) as diagonal, with the non-zero parameters representing the hydrostatic stiffness.

- The matrix N, shown in Fig. 5-(b), is given by:

$\mathbf{N}=\left(m+\mu_{\infty}\right) \mathbf{D}+\mathbf{R}$.

- The differential matrix $\mathbf{D}$ is shown in Fig. 5-(c). The first column is zero since the first basis function is a constant and its derivative is zero. Likewise, the parameter values in the upper left and lower right quadrants are zero, since the derivate of a cosine is a sine and vice-versa. The parameter values in the upper right and lower left quadrants, which transform the cosines to sines, and vicevera, can be seen to contain a structured pattern, with very large values that dominate the resulting parameter values in the matrix $\mathbf{N}$.

- The radiation matrix, R, Fig. 5-(d), contains non-zero parameter values along the main diagonal, which correspond to the radiation damping force due to the WEC velocity. The nonzero terms along the small diagonals, in the upper right and lower left quadrants, correspond to the added mass terms that are in quadrature with the velocity, whereby a cosine velocity gives a sine radiation term (and vice-versa) relating to an added mass force.
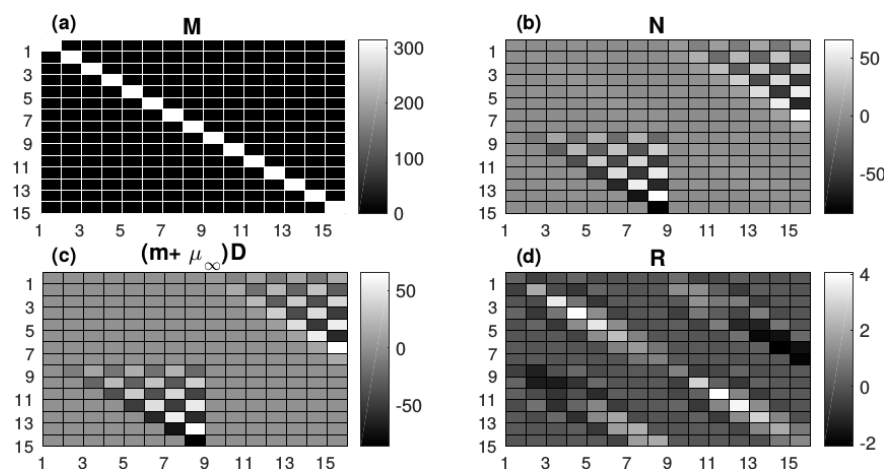

Fig. 5. Initialisation of the $M$ and $N$ matrices, where $\mathbf{N}=\left(m+\mu_{\infty}\right) \mathbf{D}+\mathbf{R}$
3) Control settings: The value for the various control parameters employed in the test cases are listed in Table I. The collocation points are displayed in Fig. 6, employing a sinusoidal spacing over the control horizon, to minimise any Gibbs phenomena, as discussed in [13].

TABLE I

Control SETting VALUES USED IN THE TEST CASE EXAMPLES.

\begin{tabular}{|l|l|l|}
\hline Parameter & Symbol & Value \\
\hline Control horizon & $T_{0}$ & $2 s$ \\
RLS update period & $\Delta t_{R L S}$ & $0.2 \mathrm{~s}$ \\
Optimal trajectory update period & $\Delta t_{R H P C}$ & $1 \mathrm{~ms}$ \\
RLS forgetting factor & $\lambda$ & 0.995 \\
P initialisation & $p_{0}$ & 1 \\
Number of basis functions & $N$ & 15 \\
Geometrical constraints & $H$ & $0.1 \mathrm{~m}$ \\
\hline
\end{tabular}

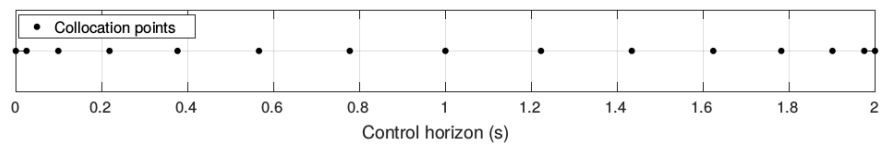

Fig. 6. The $2 N+1$ collocation points spanning the control horizon.

4) Tests: Three tests are performed for the preliminary assessment of the ARHPC, Tests 1 and 2 investigate the adaptive properties of the control model, and then Test 3 assesses the performance of the ARHPC, as follows:

Test 1: The first test examines the ARHPC model parameter adaptation, over a period of $30 \mathrm{~s}$, in the linear model and CFD NWT simulations. The RLS algorithm will be initialised at $t=3 \mathrm{~s}$, and completes 135 update steps in the remaining $27 s$. The ARHPC model parameters in the linear model simulation are expected to remain fairly constant, since both models are linear and their parameters are initialised using the same BEM hydrodynamic data. Conversely, for the CFD simulations, the ARHPC model parameters should adapt in response to the nonlinear effects captured by the CFD simulation but neglected in the ARHPC model initialisation.

Test 2: To further investigate the adaptive properties of the ARHPC, a linear simulation is performed with double the amount of hydrodynamic radiation, $2 K_{r}(t)$, to examine how the parameters in the $\mathbf{N}$ matrix respond. The importance of accurate control model estimation of the resistive forces, in optimal control calculations, is discussed theoretically in [33] and demonstrated experimentally in [16]. The fast linear simulation runtimes allows the evolution and convergence of the parameter values to be examined over a period of $100 \mathrm{~s}$.

Test 3: The final test assesses the ARHPC performance, using the CFD NWT as an evaluation tool. The importance of evaluating EMCs for WECs in a realistic simulation environment, such as CFD, is discussed in [8]. The resulting WEC motion, PTO forces and energy capture, from the CFD simulation in Test 1 , are compared against a second CFD simulation of the same controller employing non-adaptive model parameters. 


\section{Results}

1) Test 1: The total changes to the ARHPC model parameters, at $t=30 \mathrm{~s}$, are shown in Fig 7 . The changes to the model parameters in the CFD simulation, $\Delta \mathbf{M}_{\mathbf{C F D}}$ and $\Delta \mathbf{N}_{\mathbf{C F D}}$, are displayed in Figs. 7-(a) and (b) respectively, and the changes to the model parameter in the linear simulation, $\Delta \mathbf{M}_{\mathbf{L S}}$ and $\Delta \mathbf{N}_{\mathbf{L S}}$, are displayed in Figs. 7-(c) and (d) respectively.

The largest parameters changes in $\Delta \mathbf{M}_{\mathbf{C F D}}$ are seen to occur to the first few diagonal entries of the upper left and lower right quadrants, corresponding to $S_{h}$ and frequencies less than or equal to the peak wave period. Fig. 8 plots the evolution of the first three diagonal entries, $\mathbf{M}(1,1), \mathbf{M}(2,2)$ and $\mathbf{M}(3,3)$, showing a decrease in the parameter values identified from the CFD simulation. A decrease in the adaptive model representation of $S_{h}$ makes sense physically, considering $S_{h}$ for the sphere is maximal at equilibrium, and the adaptive model parameters therefore update to a linear average of the reduced $S_{h}$ values while the sphere is away from equilibrium. For comparison, the first three diagonal entries of $\mathbf{M}_{\mathbf{L S}}$ are also plotted in Fig. 8, and can be seen to remain fairly constant, as expected.

The diagonal entries of $\Delta \mathbf{N}_{\mathbf{C F D}}$, corresponding to the hydrodynamic radiation force, are seen to have changed due to the adaptive algorithm. The evolution of first three diagonal entries, $\mathbf{N}(1,1), \mathbf{N}(2,2)$ and $\mathbf{N}(3,3)$, are also plotted in Fig. 8, where the parameter values identified from the CFD simulation are seen to increase during adaptation. Due to viscous damping forces present in the CFD simulation, in addition to the radiation forces, the adaptive model increases its linear representation of the total velocity dependent forces. Once again, by comparison, the model parameters from the linear simulation are seen to remain fairly constant. However, in this case, the parameters for the velocity dependent forces in the linear simulation, $\Delta \mathbf{N}_{\mathbf{L S}}$, are seen to vary more than for the position related forces $\Delta \mathbf{M}_{\mathbf{L S}}$, likely due to this mismatch in parameterisation of the radiation convolution integral in the linear simulation and the RHPC model. Increasing the number of basis functions may reduce these small variations.

The relatively large changes around the first few diagonal parameters in the upper right and lower left quadrants of $\Delta \mathbf{N}_{\mathbf{C F D}}$, correspond to differences in the added mass effects within the CFD simulation compared to the linear model representation. Comparison of Figs. 5-(d) and 7-(b), shows that the positive values around the diagonal of the upper right quadrant of $\mathbf{R}$ become more positive in the identified model, and likewise, the negative values in the lower left quadrant become more negative, indicating an overall increase in the added mass due to the parameter adaptation. This is consistent with the findings in [18], where both the radiation and added mass values identified from CFD NWT experiments were seen to increase relative to those identified from BEM data. The evolution of $\mathbf{N}(2,9), \mathbf{N}(2,10)$ and $\mathbf{N}(3,10)$ are also plotted in Fig. 8, showing an increase in the parameter values identified from the CFD simulation, and little change to those identified from the linear simulation.
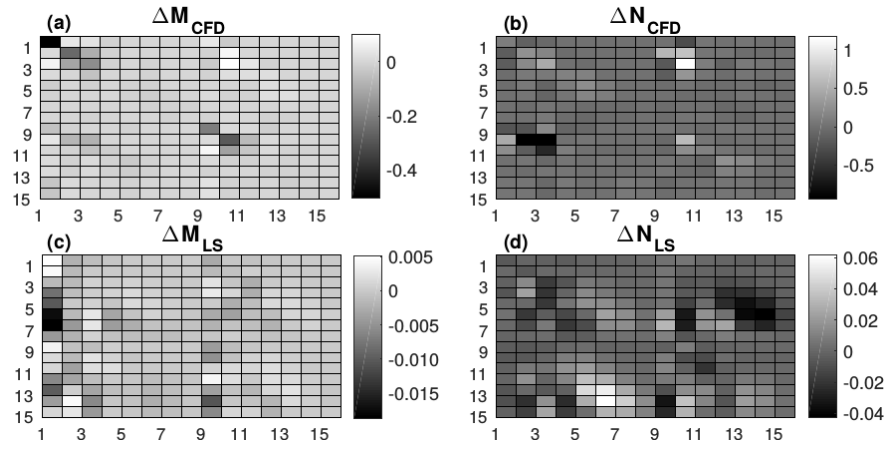

Fig. 7. Changes to parameters in the $\mathbf{M}$ and $\mathbf{N}$ matrices for the CFD simulation, (a) and (b), respectively. Changes to parameters in the $\mathbf{M}$ and $\mathbf{N}$ matrices for the linear simulation, (c) and (d), respectively.
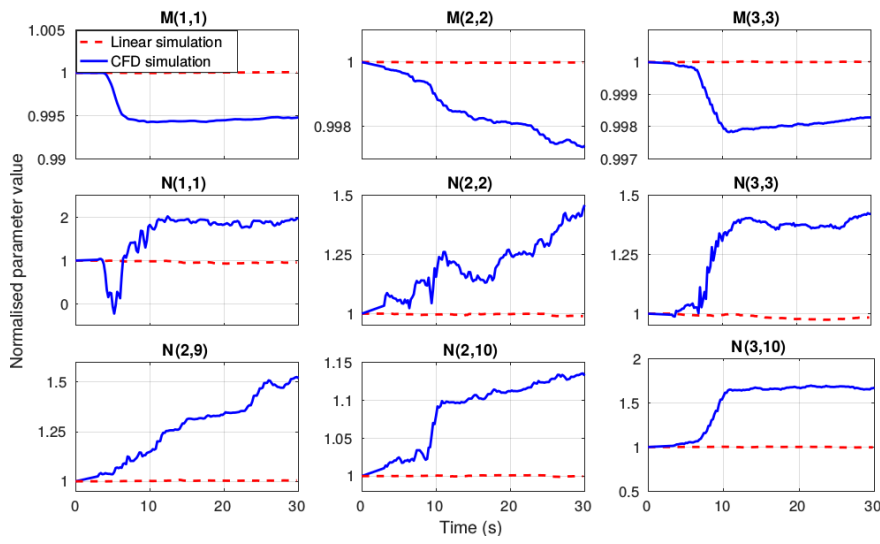

Fig. 8. Evolution of the model parameters for the linear and CFD simulations.

2) Test 2: The resulting parameter adaptation due to the doubling of the hydrodynamic radiation force in the linear simulation is illustrated in Fig 9-(a), which plots the evolution of the first three diagonal parameter values of the $\mathbf{N}$ matrix. Fig 9-(a) shows that the parameters $\mathbf{N}(1,1), \mathbf{N}(2,2)$ and $\mathbf{N}(3,3)$, which relate to the hydrodynamic radiation, converge to values approximately double their initial values, as might be expected. The effect of the adaptation of all the parameters in the $\mathbf{N}$ matrix is demonstrated in Fig 9-(b), which plots the actual simulated radiation force over a control horizon, and the linear control model approximation to this at the 15 collocation points, for both the initial and the adapted control models. Fig 9-(b) shows that the initial model approximation is quite poor, with values about half the simulated radiation force, whereas the updated model manages to approximate the simulated radiation force well.
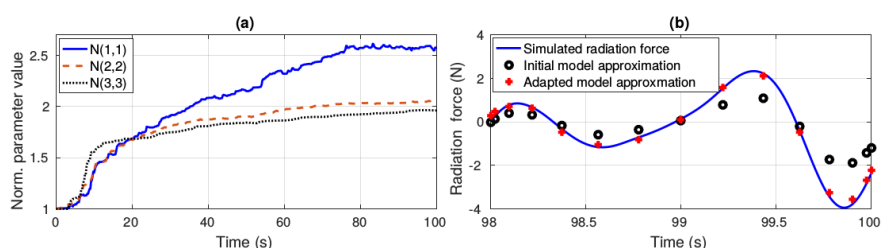

Fig. 9. (a) Evolution of the model parameters for the linear simulation with double the radiation force (b) The simulated radiation force and the control model approximation using the initial and adapted $(t=100 \mathrm{~s})$ parameter values 
3) Test 3: The performance of the ARHPC, versus its nonadaptive counterpart, is shown in Figs. 10-12. The WEC displacement and energy absorption are plotted in Fig. 10, showing the model adaptation leads to the ARHCP calculating an optimal trajectory with smaller amplitude displacements, yet yielding more energy than the nonadaptive controller.

For this particular case study, the real system had more viscous drag than in the initial RHPC model, and as a result the adaptation slows down the system response to reduce viscous drag losses. However, if the initial RHPC model overspecified the amount of viscous drag, then the adaptation would speed up the response. The correct nature and magnitude of this filtering effect, which speeds or slows the system response, is not known a priori and will vary as the input wave conditions and system characteristics change over time. The use of an adaptive model, allows the control to determine the appropriate system response, by regularly updating and improving its description of the real system, based on online measurements.
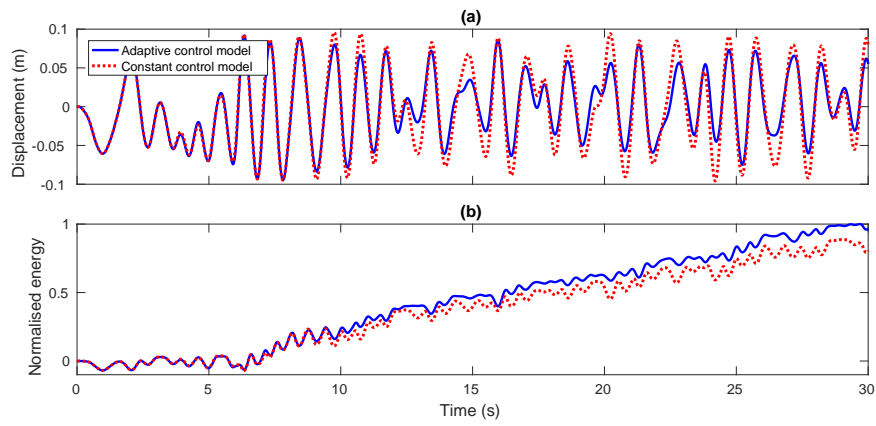

Fig. 10. Results of the adaptive RHPC versus the constant RHPC. (a) the WEC displacement, and (b) the energy absorbed by the PTO.

In addition to smaller displacements, the PTO forces applied to the WEC by the ARHPC controller are also smaller than those applied by the nonadaptive controller, as shown in Fig. 11. The optimal PTO force calculated by the RHPC and the actual PTO force applied to the WEC, are plotted in for the constant control model case (a) and the ARHPC (b). The mismatch between the optimal and actual PTO force is due to the trajectory tracking of the backstepping control which ensures the WEC follows the optimal velocity trajectory. Fig. 11 shows that, as the ARHPC model adapts and better represents the actual WEC dynamics in the CFD NWT, the calculated PTO force and the actual PTO force converge.

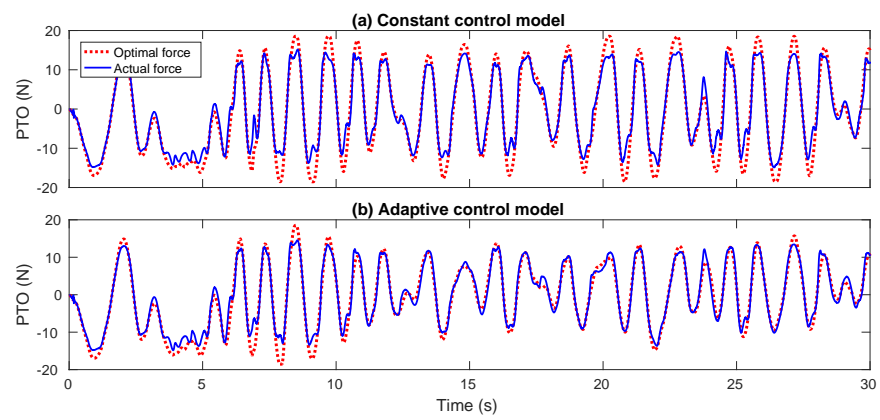

Fig. 11. The calculated optimal PTO force trajectory and the actual PTO force applied to the WEC after the backstepping control in the CFD simulation.
Another advantage of the ARHPC, over its nonadaptive counterpart, is illustrated in Fig. 12, which plots the amount of energy flowing in and out of the PTO, normalised against the total absorbed energy. Fig. 12-(a) shows that, for the WEC with the constant control model, about 4.5 times the absorbed energy came into the PTO from the WEC and 3.5 times from the PTO to the WEC. However, for the WEC with the adaptive control model, Fig. 12-(b) only 3 times the total absorbed energy came into the PTO from the WEC and 2 times from the PTO to the WEC. Fig. 12-(c) shows the ratio of energy in to energy out for the two controllers, showing the improvement of the adaptive controller over the constant controller once the adaption begins. Limiting the amount of bi-directional power flow is important when generator inefficiencies are considered, due to energy losses occurring every time energy is transferred. Additionally, the smaller bi-directional power flow results in a reduction of the PTO power capacity requirement and associated capital costs.
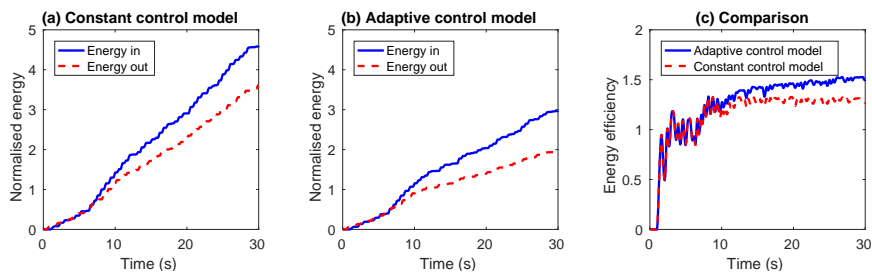

Fig. 12. The flow of energy to and from the PTO, normalised against the total absorbed energy, for the (a) constant control model and (b) the adaptive control model. (c) The ratio of energy in to energy out, as a measure of the energy efficiency of the two controllers.

Regarding computational demand, on an Intel Xeon CPU E5-2450v2@2.5 GHz, the unoptimised, MATLAB interpreted control algorithm required $0.04 \mathrm{~s}$ for backstepping, $0.5 \mathrm{~s}$ for optimal trajectory calculation and $0.15 \mathrm{~s}$ for parameter update. This is already fast enough for real-time control of full scale WECs, where recalculation of the optimal trajectories is typically required on a second-by-second basis. With optimised and compiled code, we would expect roughly an order of magnitude increase in computational speed, therefore real-time control seems feasible with the ARHPC.

\section{CONCLUSION}

The optimal controller presented in this paper, the ARHPC, utilises a linear control model which results in a quadratic optimisation problem that lends itself to efficient optimisation routines, enabling the possibility of real-time control. Typical weaknesses of a linear WEC model, such as limited range of validity, are overcome by employing system identification techniques to update the model parameters online so that the linear model remains representative in changing sea conditions and WEC states.

The initial assessment of the ARHPC, performed in a highfidelity CFD simulation environment, gives promising results, with the ARHPC absorbing more energy than its non adaptive counterpart. In addition to producing more power, the resulting WEC displacements and PTO forces from the ARHPC were smaller than from the nonadaptive controller, which would 
reduce the required capital and operational costs for the WEC. Both controllers were evaluated under the assumption of perfect knowledge of the incident wave series over the control horizon and assuming the PTO to be $100 \%$ efficient. Another advantage displayed by the ARHCP, was the lower amount of bi-directional power flow required to/from the PTO, which decreases the amount of energy loss when PTO inefficiencies are considered.

\section{ACKNOWLEDGMENTS}

This paper is based upon work supported by Science Foundation Ireland under Grant No. 13/IA/1886.

\section{REFERENCES}

[1] J. Scruggs, S. Lattanzio, A. Taflanidis, and I. Cassidy, "Optimal causa control of a wave energy converter in a random sea," Applied Ocean Research, vol. 42, no. 0, pp. 1 - 15, 2013.

[2] J. Falnes, Ocean Waves and Oscillating Systems. Cambridge, UK Cambridge University Press, 2002.

[3] U. Korde and J. Ringwood, Hydrodynamic control of wave energy devices. Cambridge University Press, New York, 2016.

[4] J. Hals, J. Falnes, and T. Moan, "A comparison of selected strategies for adaptive control of wave energy converters," Journal of Offshore Mechanics and Arctic Engineering, vol. 133, no. 3, 2011.

[5] G. Bacelli and J. V. Ringwood, "Numerical optimal control of wave energy converters," IEEE Transactions on Sustainable Energy, vol. 6 , no. 2, pp. 294-302, 2015

[6] W. Cummins, "The impulse response function and ship motions," Tech Rep., 1962.

[7] G. Giorgi, M. Penalba, and J. Ringwood, "Nonlinear Froude-Krylov force representations for heaving buoy wave energy converters," in Proceedings of the $3^{\text {rd }}$ Asian Wave and Tidal Energy Conference (AWTEC), 2016.

[8] J. Davidson, C. Windt, G. Giorgi, R. Genest, and J. Ringwood, OpenFOAM - Selected papers from the 11th Workshop. Springer, 2017, ch. Evaluationg of energy maximising control systems for wave energy converters using OpenFOAM.

[9] G. Giorgi, M. Penalba, and J. Ringwood, "Nonlinear hydrodynamic force relevance for different wave energy converter types," in Proceedings of the $3^{\text {rd }}$ Asian Wave and Tidal Energy Conference (AWTEC), 2016.

[10] S. R. Nielsen, Q. Zhou, M. M. Kramer, B. Basu, and Z. Zhang, "Optimal control of nonlinear wave energy point converters," Ocean Engineering, vol. 72, pp. 176-187, 2013.

[11] G. Bacelli, R. Genest, and J. Ringwood, "Nonlinear control of a flaptype wave energy converter with a non-ideal power take-off system," IFAC Annual Reviews in Control, vol. 40, pp. 116-126, 2015.

[12] M. Richter, M. Magana, O. Sawodny, and T. Brekken, "Nonlinear mode predictive control of a point absorber wave energy converter," IEEE Transactions on Sustainable Energy, vol. 4, no. 1, pp. 118-126, 2013.

[13] R. Genest and J. V. Ringwood, "A critical comparison of modelpredictive and pseudospectral control for wave energy devices," Journal of Ocean Engineering and Marine Energy, pp. 1-15, 2016.

[14] J. Davidson and J. Ringwood, "Mathematical modelling of mooring systems for wave energy converters - a review," Energies, 2017.

[15] E. Anderlini, D. I. Forehand, P. Stansell, Q. Xiao, and M. Abusara, "Control of a point absorber using reinforcement learning," IEEE Transactions on Sustainable Energy, vol. 7, no. 4, pp. 1681-1690, 2016.

[16] J. Davidson, R. Genest, and J. V. Ringwood, "Adaptive control of a wave energy converter simulated in a numerical wave tank," in Proceedings of the 12th European Wave and Tidal Energy Conference, 2017.

[17] J. V. Ringwood, J. Davidson, and S. Giorgi, Numerical Modeling of Wave Energy Converter: State-of-the-art techniques for single WEC and converter arrays. Elsevier, 2016, ch. Identifying models using recorded data.

[18] J. Davidson, S. Giorgi, and J. V. Ringwood, "Linear parametric hydrodynamic models for ocean wave energy converters identified from numerical wave tank experiments," Ocean Engineering, vol. 103, pp. $31-39,2015$

[19] S. Giorgi, J. Davidson, and J. V. Ringwood, "Identification of wave energy device models from numerical wave tank data - part 2: Databased model determination," IEEE Transactions on Sustainable Energy, vol. 7, no. 3, pp. 1020-1027, 2016.
[20] J. Davidson, S. Giorgi, and J. V. Ringwood, "Identification of wave energy device models from numerical wave tank data - part 1: Numerical wave tank identification tests," IEEE Transactions on Sustainable Energy, vol. 7, no. 3, pp. 1012-1019, 2016.

[21] R. Genest and J. V. Ringwood, "A critical comparison of modelpredictive and pseudospectral control for wave energy devices," Journal of Ocean Engineering and Marine Energy, pp. 1-15, 2016.

[22] D. Huybrechs, "On the fourier extension of nonperiodic functions," SIAM Journal on Numerical Analysis, vol. 47, no. 6, pp. 4326-4355, 2010.

[23] M. H. Hayes, "9.4: Recursive least squares," Statistical Digital Signal Processing and Modeling, p. 541, 1996.

[24] B. Orel and A. Perne, "Computations with half-range chebyshev polynomials," Journal of Computational and Applied Mathematics, vol. 236, no. 7, pp. 1753-1765, 2012

[25] J. Mattingley, Y. Wang, and S. Boyd, "Receding horizon control," IEEE Control Systems, vol. 31, no. 3, pp. 52-65, 2011.

[26] I. Kanellakopoulos, P. V. Kokotovic, and A. S. Morse, "Systematic design of adaptive controllers for feedback linearizable systems," IEEE Transactions on Automatic control, vol. 36, no. 11, pp. 1241-1253, 1991.

[27] F. Fusco and J. V. Ringwood, "Hierarchical robust control of oscillating wave energy converters with uncertain dynamics," IEEE Transactions on Sustainable Energy, vol. 5, no. 3, pp. 958-966, 2014.

[28] — - "Short-term wave forecasting for real-time control of wave energy converters," IEEE Transactions on Sustainable Energy, vol. 1, no. 2, pp. 99-106, 2010

[29] A. Babarit and G. Delhommeau, "Theoretical and numerical aspects of the open source bem solver nemoh," in 11th European Wave and Tidal Energy Conference (EWTEC2015), 2015.

[30] J. Davidson, M. Cathelain, L. Guillemet, T. Le Huec, and J. Ringwood, "Implementation of an openfoam numerical wave tank for wave energy experiments," in Proceedings of the 11th European Wave and Tidal Energy Conference, 2015.

[31] G. Giorgi and J. V. Ringwood, "Implementation of latching control in a numerical wave tank with regular waves," Journal of Ocean Engineering and Marine Energy, vol. 2, no. 2, pp. 211-226, 2016.

[32] P. Schmitt and B. Elsäßer, "The application of froude scaling to model tests of oscillating wave surge converters," Ocean Engineering, vol. 141, pp. 108-115, 2017.

[33] A. Merigaud and J. Ringwood, "Optimal trajectories, nonlinear models and constraints in wave energy device control," in International Federation of Automatic Control (IFAC), 2017.

Romain Genest received the bachelors degrees in mechanics and electronics from the cole Normale Suprieure de Rennes, France, the masters degree in hydrodynamics and the $\mathrm{Ph} . \mathrm{D}$. degree in mechanics from the cole Centrale de Nantes, France, and the Agregation diploma in engineering sciences to teach in French Preparatory School. His Ph.D. thesis concerned the development and experimental validation of control strategies for WECs. He is currently a PostDoctoral Researcher with the Centre for Ocean Energy Research (COER), Maynooth University, Ireland, where he focuses on the control of WECs.

Josh Davidson received his Bachelors Degree in Physical Sciences in 2006 at James Cook University, Australia, with first class honours. Josh then studied a $\mathrm{PhD}$ at James Cook University, focussing on energy harvesting for marine based sensors, and was co-funded by the Commonwealth Scientific and Industrial Research Organisation's (CSIRO) Energy Technology Division. Josh currently works at the COER, Maynooth University, Ireland, where his research explores nonlinear modelling and control of WECs.

John Ringwood received the Diploma in Electrical Engineering from Dublin Institute of Technology in 1981 and the $\mathrm{PhD}$ (in Control Systems) from Strathclyde University, Scotland in 1985. He is currently Professor of Electronic Engineering (EE) at Maynooth University, and Director of COER. He was Head of the EE Department at NUI Maynooth from 2000 until 2005 , developing the Department from a greenfield site. John's research interest cover time series modelling, wave energy and biomedical engineering. $\mathrm{He}$ is a Chartered Engineer and a Fellow of the Institution of Engineers of Ireland. 